International Journal of Pure and Applied Mathematics

Volume 112 No. 4 2017, 655-671

ISSN: 1311-8080 (printed version); ISSN: 1314-3395 (on-line version)

url: http://www.ijpam.eu

doi: 10.12732/ijpam.v112i4.1

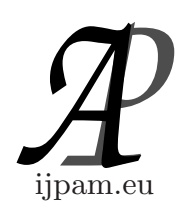

\title{
DERIVATIONS AND CENTROIDS OF FOUR-DIMENSIONAL ASSOCIATIVE ALGEBRAS
}

\author{
A.O. Abdulkareem ${ }^{1}$, M.A. Fiidow ${ }^{2}$, I.S. Rakhimov ${ }^{3}$ § \\ ${ }^{1}$ Department of Mathematics \\ College of Physical Sciences \\ Federal University of Agriculture Abeokuta \\ PMB 2240, Alabata road, Abeokuta, Ogun State, NIGERIA \\ ${ }^{2,3}$ Department of Mathematics \\ Faculty of Science \\ Universiti Putra Malaysia, UPM 43400 Serdang \\ Selangor Darul Ehsan, MALAYSIA \\ ${ }^{2,3}$ Institute for Mathematical Research (INSPEM) \\ Universiti Putra Malaysia, UPM 43400 Serdang \\ Selangor Darul Ehsan, MALAYSIA
}

\begin{abstract}
In the paper derivations and centroids of four-dimensional associative algebras are described. We also identify the class of algebras called characteristically nilpotent among four-dimensional associative algebras.
\end{abstract}

AMS Subject Classification: 16D70

Key Words: derivation, centroid, associative algebra, characteristically nilpotent

Received: $\quad$ March 9, 2016

Revised: $\quad$ November 10, 2016

Published: $\quad$ February 13, 2017

$\S_{\text {Correspondence author }}$ (c) 2017 Academic Publications, Ltd. url: www.acadpubl.eu 


\section{Introduction}

The interest in the study of derivations of algebras goes back to a paper by Jacobson [1]. There, Jacobson proved that any Lie algebra over a field of characteristic zero which has non degenerate derivations is nilpotent. In the same paper, he asked for the converse. Dixmier and Lister [2] have given a negative answer to the converse of Jacobson's hypothesis by constructing an example of a nilpotent Lie algebra all of whose derivations are nilpotent (hence degenerate). Lie algebras whose derivations are nilpotent have been called characteristically nilpotent. The result of Dixmier and Lister in [2] is assumed to be the origin of the theory of characteristically nilpotent Lie algebras. A few years later in 1959, Leger and Togo [3] published a paper showing the importance of the characteristically nilpotent Lie algebras. The theory of characteristically nilpotent Lie algebras constitutes an independent research object since 1955. Until then, most studies about Lie algebras were oriented to the classical aspects of the theory, such as semi-simple and reductive Lie algebras (see [4]).

The structural theory of finite dimensional associative algebras have been treated by [5]. Many interesting results related to the problem have appeared since then. Further works in this field can be found in [6], [7], [8], [9] and [10]. Further development of the theory of associative algebras was in 80-s of the last century when many open problems, remaining on unsolved since 30-s, have been solved

Centroids of algebras play an important role in the classification problems and in different areas of structure theory of algebras. The centroid of some classes of a Lie algebra is known to be a field and this fact plays an important role in the classification problem of the class considered(see [11]). Benkart and Neher studied extended affine and root graded Lie algebras in [11]. Melville in [12] studied the centroids of nilpotent Lie algebra. Centroids and derivations of associative algebras in dimension less than four has been treated in [13] and provided an impetus to further the study in higher dimension.

In this study, we concentrate on the derivations and centroids of associative algebras in dimension four. Using the classification result of associative algebras, we give a description of derivations and centroids of associative algebras in dimension 4. 


\section{Preliminaries}

In this section we review some elementary feats on algebras, derivations and centroids.

Definition 1. An algebra $A$ over a field $\mathbb{K}$ is a vector space over $\mathbb{K}$ equipped with a bilinear map

$$
\lambda: A \times A \rightarrow A .
$$

Definition 2. An associative algebra $A$ is an algebra with bilinear map $\lambda: A \times A \longrightarrow A$ satisfying the associative law:

$$
\lambda(\lambda(x, y), z)=\lambda(x, \lambda(y, z)) \text { for all } x, y, z \in A
$$

Definition 3. A Lie algebra $L$ over a field $\mathbb{K}$ is an algebra satisfying the following conditions:

$$
\begin{gathered}
\lambda(x, x)=0, \\
\lambda(\lambda(x, y), z)+\lambda(\lambda(y, z), x)+\lambda(\lambda(z, x), y)=0,
\end{gathered}
$$

for all $x, y, \in L$.

It is customary to denote the binary operation $\lambda$ in Lie algebras case as the bracket $[\cdot, \cdot]$.

Definition 4. Let $\left(A_{1}, \cdot\right)$ and $\left(A_{2}, \bullet\right)$ be two associative algebras over a field $\mathbb{K}$. A homomorphism from $A_{1}$ to $A_{2}$ is a $\mathbb{K}$-linear mapping $f: A_{1} \longrightarrow A_{2}$ such that

$$
f(x \cdot y)=f(x) \bullet f(y), \text { for all } x, y \in A_{1} .
$$

The set of all homomorphisms from $A_{1}$ to $A_{2}$ is denoted by $\operatorname{Hom}_{\mathbb{K}}\left(A_{1}, A_{2}\right)$. A bijective homomorphism is called isomorphism and the corresponding algebras are said to be isomorphic. If $A_{1}=A_{2}=A$, then $\operatorname{Hom}_{\mathbb{K}}(A)$ is an associative algebra with respect to the composition operation. The algebra $\operatorname{Hom}_{\mathbb{K}}(A)$ is called the algebra of endomorphisms of $A$.

Example 5. Let $(A, \cdot)$ be an associative algebra. Define the product of two elements in $A$ as follows:

$$
[a, b]=a \cdot b-b \cdot a .
$$

Then $(A,[\cdot, \cdot])$ is a Lie algebra. Particularly, the associative algebra $\operatorname{Hom}_{\mathbb{K}}(A)$ above is a Lie algebra under $[f, g]=f \circ g-g \circ f$. 
Definition 6. A linear transformation $d$ of an associative algebra $(A, \cdot)$ is called a derivation if for any $x, y \in A$ one has

$$
d(x \cdot y)=d(x) \cdot y+x \cdot d(y)
$$

The set of all derivations of an associative algebra $A$ is denoted by $\operatorname{Der}(A)$.

In Lie algebras case the property to be characteristically nilpotent is an important property. For associative algebras case this property is defined similarly to that of Lie algebras case.

The following facts can easily be proven.

Proposition 7. Let $A$ be an algebra. Then $\operatorname{Der}(A)$ is a Lie algebra with respect to the bracket $[f, g]=f \circ g-g \circ f$.

For $a \in A$, let $L_{a}$ and $R_{a}$ be the elements of $\operatorname{Hom}_{\mathbb{K}}(A)$ defined by

$$
L_{a}(x)=a \cdot x, \quad R_{a}(x)=x \cdot a, \text { for } x \in A .
$$

Theorem 8. Let $d \in \operatorname{Hom}_{\mathbb{K}}(A)$. Then the following conditions are equivalent :

1. $d \in \operatorname{Der}(A)$,

2. $\left[d, L_{x}\right]=L_{d(x)}, \quad x \in A$,

3. $\left[d, R_{y}\right]=R_{d(y)}, \quad y \in A$.

Proof. The proof is straightforward.

Definition 9. Let $A$ be an arbitrary associative algebra over a field $\mathbb{K}$ : The centroid of $\Gamma(A)$, is defined by

$$
\Gamma(A)=\{\phi \in \operatorname{End}(A): \phi(x \cdot y)=\phi(x) \cdot y=x \cdot \phi(y)\} .
$$

Definition 10. Let $H$ be a non empty subset of $A$. The set

$$
Z_{A}(H)=\{x \in A: x \cdot H=H \cdot x=0\} .
$$

is said to be centralizer of $H$ in $A$.

It must however be noted that $Z_{A}(A)=Z(A)$, the center of $A$.

Definition 11. Let $\phi \in \operatorname{End}_{\mathbb{K}}(A)$. If $\phi(A) \subseteq Z(A)$ and $\phi\left(A^{2}\right)=0$, then $\phi$ is called a central derivation.

The set of all central derivations of $A$ is denoted by $C(A)$.

In the foregoing, we give a few earlier results on some properties of centroids of associative algebras which show the relationship between derivation and centroid. The proof of some of the facts given below can be found in [13]. 
Proposition 12. Let $A$ be an associative algebra over a field $\mathbb{K}$. Then

1. If $\phi \in \Gamma(A)$ and $d \in \operatorname{Der}(A)$ then $\phi \circ d$ is a derivation of $A$.

2. $C(A)=\Gamma(A) \cap \operatorname{Der}(A)$.

3. For any $d \in \operatorname{Der}(A)$ and $\phi \in \Gamma(A)$ one has

- The composition $d \circ \phi$ is in $\Gamma(A)$ if and only if $\phi \circ d$ is a central derivation of $A$;

- The composition $d \circ \phi$ is a derivation of $A$ if and only if $[d \circ \phi]$ is a central derivation of $A$.

\section{Procedure for finding derivations}

Let $A$ be an $n$-dimensional associative algebra and $d$ be its derivation. Fix a basis $\left\{e_{1}, e_{2}, \ldots, e_{n}\right\}$ of $A$. Then,

$$
d \circ L_{e_{\mathrm{i}}}(y)=L_{d\left(e_{\mathrm{i}}\right)}(y)+L_{e_{\mathrm{i}}} \circ d(y),
$$

for basis vectors $\left\{e_{1}, e_{2}, \ldots, e_{n}\right\}$, i.e.,

$$
\left(d \circ L_{e_{\mathrm{i}}}-L_{e_{\mathrm{i}}} \circ d\right)(y)=L_{d\left(e_{\mathrm{i}}\right)}(y) .
$$

The derivation $d$ being a linear transformation of the vector space $A$ is represented in a matrix form $\left(d_{i j}\right)_{i, j=1,2, \cdots, n}$ i.e., $d\left(e_{i}\right)=\sum_{j=1}^{n} d_{j i} e_{j}, \quad i=1,2, \cdots, n$. Hence.

$$
\begin{gathered}
\left(d \circ L_{e_{\mathrm{i}}}-L_{e_{\mathrm{i}}} \circ d\right)(y)=\sum_{j=1}^{n} d_{j i} L_{e_{\mathrm{j}}}(y) \\
d \circ L_{e_{\mathrm{i}}}-L_{e_{\mathrm{i}}} \circ d=\sum_{j=1}^{n} d_{j i} L_{e_{\mathrm{j}}}
\end{gathered}
$$

for all $i=1,2, \cdots, n$.

The last system of equations along with structure of $A$ gives constraints for elements of the matrix $d$. Solving the system of equations, we find the matrix

$$
d=\left(d_{i j}\right)=\left(\begin{array}{cccc}
d_{11} & d_{12} & \cdots & d_{1 n} \\
\vdots & \vdots & \vdots & \vdots \\
d_{n 1} & d_{n 2} & \cdots & d_{n n}
\end{array}\right)
$$


As mention earlier, we provide classification results of four-dimensional associative algebras from [10] which we make use in our study. Recall that $m^{\text {th }}$ isomorphism class of associative algebras in dimension $n$ is denoted by $A s_{n}^{m}$

\subsection{Four-dimensional Associative algebras}

Theorem 13. Any four-dimensional complex associative algebra can be included in one of the following isomorphism classes of algebras:

$$
\begin{aligned}
& A s_{4}^{1}: e_{1} e_{1}=e_{3}, \quad e_{2} e_{2}=e_{4} ; \\
& A s_{4}^{2}: e_{1} e_{2}=e_{3}, \quad e_{2} e_{1}=e_{4} \text {; } \\
& A s_{4}^{3}: e_{1} e_{2}=e_{4}, \quad e_{3} e_{1}=e_{4} \text {; } \\
& A s_{4}^{4}: e_{1} e_{1}=e_{4}, \quad e_{2} e_{2}=e_{2}, \quad e_{2} e_{3}=e_{3} \\
& A s_{4}^{5}: e_{1} e_{1}=e_{4}, \quad e_{2} e_{2}=e_{2}, \quad e_{3} e_{2}=e_{3} \text {; } \\
& A s_{4}^{6}: e_{1} e_{2}=e_{3}, \quad e_{2} e_{1}=e_{4}, \quad e_{2} e_{2}=-e_{3} \text {; } \\
& A s_{4}^{7}: e_{1} e_{2}=e_{3}, \quad e_{2} e_{1}=-e_{3}, \quad e_{2} e_{2}=e_{4} \text {; } \\
& A s_{4}^{8}: e_{1} e_{2}=e_{4}, \quad e_{2} e_{1}=-e_{4}, \quad e_{3} e_{3}=e_{4} \text {; } \\
& A s_{4}^{9}(\alpha): e_{1} e_{2}=e_{4}, \quad e_{2} e_{1}=\frac{1+\alpha}{1-\alpha} e_{4}, \quad e_{2} e_{2}=e_{3}, \alpha \in \mathbb{C} \backslash\{1\} ; \\
& A s_{4}^{10}: e_{1} e_{1}=e_{1}, \quad e_{1} e_{2}=e_{2}, \quad e_{1} e_{3}=e_{3}, \quad e_{1} e_{4}=e_{4} ; \\
& A s_{4}^{11}: e_{1} e_{1}=e_{1}, \quad e_{1} e_{2}=e_{2}, \quad e_{1} e_{3}=e_{3}, \quad e_{4} e_{1}=e_{4} \text {; } \\
& A s_{4}^{12}: e_{1} e_{1}=e_{1}, \quad e_{1} e_{2}=e_{2}, \quad e_{1} e_{4}=e_{4}, \quad e_{2} e_{3}=e_{4} \text {; } \\
& A s_{4}^{13}: e_{1} e_{1}=e_{1}, \quad e_{1} e_{2}=e_{2}, \quad e_{3} e_{3}=e_{3}, \quad e_{3} e_{4}=e_{4} \text {; } \\
& A s_{4}^{14}: e_{1} e_{1}=e_{1}, \quad e_{1} e_{4}=e_{4}, \quad e_{2} e_{1}=e_{2}, \quad e_{2} e_{4}=e_{3} \text {; } \\
& A s_{4}^{15}: e_{1} e_{1}=e_{1}, \quad e_{1} e_{4}=e_{4}, \quad e_{2} e_{1}=e_{2}, \quad e_{3} e_{1}=e_{3} \text {; } \\
& A s_{4}^{16}: e_{1} e_{1}=e_{1}, \quad e_{2} e_{1}=e_{2}, \quad e_{3} e_{1}=e_{3}, \quad e_{4} e_{1}=e_{4} \text {; } \\
& A s_{4}^{17}: e_{1} e_{1}=e_{1}, \quad e_{2} e_{1}=e_{2}, \quad e_{3} e_{2}=e_{4}, \quad e_{4} e_{1}=e_{4} \text {; } \\
& A s_{4}^{18}: e_{1} e_{1}=e_{1}, \quad e_{2} e_{1}=e_{2}, \quad e_{3} e_{3}=e_{3}, \quad e_{4} e_{3}=e_{4} \text {; } \\
& A s_{4}^{19}: e_{1} e_{1}=e_{1}, \quad e_{2} e_{2}=e_{2}, \quad e_{2} e_{4}=e_{4}, \quad e_{3} e_{1}=e_{3} \text {; } \\
& A s_{4}^{20}: e_{1} e_{1}=e_{1}, \quad e_{2} e_{2}=e_{2}, \quad e_{3} e_{3}=e_{3}, \quad e_{4} e_{4}=e_{4} \text {; } \\
& A s_{4}^{21}: e_{1} e_{1}=e_{3}, \quad e_{1} e_{3}=e_{4}, \quad e_{2} e_{2}=-e_{4}, \quad e_{3} e_{1}=e_{4} \text {; }
\end{aligned}
$$

$A s_{4}^{22}: e_{1} e_{1}=e_{4}, \quad e_{1} e_{2}=e_{3}, \quad e_{2} e_{1}=-e_{3}, \quad e_{2} e_{2}=-2 e_{3}+e_{4}$ 
$A s_{4}^{23}(\alpha): e_{1} e_{1}=e_{4}, \quad e_{1} e_{2}=e_{3}, \quad e_{2} e_{1}=-\alpha e_{4}, \quad e_{2} e_{2}=-e_{3}, \alpha \in \mathbb{C}$ $A s_{4}^{24}: e_{1} e_{1}=e_{4}, \quad e_{1} e_{2}=e_{4}, \quad e_{2} e_{1}=-e_{4}, \quad e_{3} e_{3}=e_{4}$;

$A s_{4}^{25}: e_{1} e_{1}=e_{4}, \quad e_{1} e_{4}=-e_{3}, \quad e_{2} e_{1}=e_{3}, \quad e_{4} e_{1}=-e_{3} ;$

$A s_{4}^{26}: e_{1} e_{1}=e_{1}, \quad e_{1} e_{2}=e_{2}, \quad e_{1} e_{3}=e_{3}, \quad e_{1} e_{4}=e_{4}, \quad e_{2} e_{1}=e_{2}$;

$A s_{4}^{27}: e_{1} e_{1}=e_{1}, \quad e_{1} e_{2}=e_{2}, \quad e_{1} e_{4}=e_{4}, \quad e_{3} e_{1}=e_{3}, \quad e_{4} e_{1}=e_{4}$;

$A s_{4}^{28}: e_{1} e_{1}=e_{1}, \quad e_{1} e_{2}=e_{2}, \quad e_{2} e_{1}=e_{2}, \quad e_{3} e_{1}=e_{3}, \quad e_{4} e_{1}=e_{4}$;

$A s_{4}^{29}: e_{1} e_{1}=e_{1}, \quad e_{1} e_{3}=e_{3}, \quad e_{1} e_{4}=e_{4}, \quad e_{2} e_{2}=e_{2}, \quad e_{3} e_{2}=e_{3}$;

$A s_{4}^{30}: e_{1} e_{1}=e_{1}, \quad e_{1} e_{3}=e_{3}, \quad e_{2} e_{2}=e_{2}, \quad e_{2} e_{4}=e_{4}, \quad e_{4} e_{1}=e_{4}$;

$A s_{4}^{31}: e_{1} e_{1}=e_{1}, \quad e_{2} e_{2}=e_{2}, \quad e_{2} e_{3}=e_{3}, \quad e_{3} e_{1}=e_{3}, \quad e_{4} e_{1}=e_{4} ;$

$A s_{4}^{32}: e_{1} e_{1}=e_{1}, \quad e_{2} e_{2}=e_{2}, \quad e_{2} e_{3}=e_{3}, \quad e_{3} e_{1}=e_{3}, \quad e_{4} e_{2}=e_{4}$;

$A s_{4}^{33}: e_{1} e_{1}=e_{1}, \quad e_{2} e_{2}=e_{2}, \quad e_{3} e_{2}=e_{3}, \quad e_{4} e_{3}=e_{3}, \quad e_{4} e_{4}=e_{4}$;

$A s_{4}^{34}: e_{1} e_{1}=e_{1}, \quad e_{2} e_{2}=e_{2}, \quad e_{3} e_{3}=e_{3}, \quad e_{3} e_{4}=e_{4}, \quad e_{4} e_{3}=e_{4}$;

$A s_{4}^{35}(\alpha): e_{1} e_{1}=e_{4}, \quad e_{1} e_{2}=\alpha e_{4}, \quad e_{2} e_{1}=-\alpha e_{4}, \quad e_{2} e_{2}=e_{4}, \quad e_{3} e_{3}=e_{4}, \alpha \in \mathbb{C} ;$

$A s_{4}^{36}: e_{1} e_{1}=e_{4}, \quad e_{1} e_{4}=-e_{3}, \quad e_{2} e_{1}=e_{3}, \quad e_{2} e_{2}=e_{3}, \quad e_{4} e_{1}=-e_{3} ;$

$A s_{4}^{37}: e_{1} e_{2}=e_{4}, \quad e_{1} e_{3}=e_{4}, \quad e_{2} e_{1}=-e_{4}, \quad e_{2} e_{2}=e_{4}, \quad e_{3} e_{1}=e_{4}$;

$A s_{4}^{38}: e_{1} e_{1}=e_{1}, \quad e_{1} e_{2}=e_{2}, \quad e_{1} e_{3}=e_{3}, \quad e_{1} e_{4}=e_{4}, \quad e_{2} e_{1}=e_{2}, \quad e_{3} e_{1}=e_{3}$;

$A s_{4}^{39}: e_{1} e_{1}=e_{1}, \quad e_{1} e_{2}=e_{2}, \quad e_{1} e_{3}=e_{3}, \quad e_{1} e_{4}=e_{4}, \quad e_{3} e_{1}=e_{3}, \quad e_{3} e_{2}=e_{4}$;

$A s_{4}^{40}: e_{1} e_{1}=e_{1}, \quad e_{1} e_{2}=e_{2}, \quad e_{1} e_{3}=e_{3}, \quad e_{2} e_{1}=e_{2}, \quad e_{3} e_{1}=e_{3}, \quad e_{4} e_{1}=e_{4} ;$

$A s_{4}^{41}: e_{1} e_{1}=e_{1}, \quad e_{1} e_{2}=e_{2}, \quad e_{2} e_{1}=e_{2}, \quad e_{3} e_{3}=e_{3}, \quad e_{3} e_{4}=e_{4}, \quad e_{4} e_{3}=e_{4}$;

$A s_{4}^{42}: e_{1} e_{1}=e_{1}, \quad e_{1} e_{3}=e_{3}, \quad e_{1} e_{4}=e_{4}, \quad e_{2} e_{2}=e_{2}, \quad e_{3} e_{1}=e_{3}, \quad e_{4} e_{2}=e_{4}$;

$A s_{4}^{43}: e_{1} e_{1}=e_{1}, \quad e_{1} e_{3}=e_{3}, \quad e_{2} e_{1}=e_{2}, \quad e_{2} e_{3}=e_{4}, \quad e_{3} e_{1}=e_{3}, \quad e_{4} e_{1}=e_{4} ;$

$A s_{4}^{44}: e_{1} e_{1}=e_{1}, \quad e_{1} e_{3}=e_{3}, \quad e_{2} e_{2}=e_{2}, \quad e_{2} e_{4}=e_{4}, \quad e_{3} e_{1}=e_{3}, \quad e_{4} e_{1}=e_{4}$;

$A s_{4}^{45}: e_{1} e_{1}=e_{1}, \quad e_{1} e_{4}=e_{4}, \quad e_{2} e_{2}=e_{2}, \quad e_{2} e_{3}=e_{3}, \quad e_{3} e_{1}=e_{3}, \quad e_{4} e_{2}=e_{4} ;$

$A s_{4}^{46}: e_{1} e_{1}=e_{1}, \quad e_{2} e_{2}=e_{2}, \quad e_{2} e_{3}=e_{3}, \quad e_{2} e_{4}=e_{4}, \quad e_{3} e_{1}=e_{3}, \quad e_{4} e_{1}=e_{4}$;

$A s_{4}^{47}: e_{1} e_{1}=e_{1}, \quad e_{2} e_{2}=e_{2}, \quad e_{2} e_{3}=e_{3}, \quad e_{2} e_{4}=e_{4}, \quad e_{3} e_{2}=e_{3}, \quad e_{4} e_{2}=e_{4}$;

$A s_{4}^{48}: e_{1} e_{1}=e_{2}, \quad e_{1} e_{2}=e_{3}, \quad e_{1} e_{3}=e_{4}, \quad e_{2} e_{1}=e_{3}, \quad e_{2} e_{2}=e_{4}, \quad e_{3} e_{1}=e_{4}$;

$A s_{4}^{49}: e_{1} e_{4}=e_{2}, \quad e_{2} e_{3}=e_{2}, \quad e_{3} e_{1}=e_{1}, \quad e_{3} e_{2}=e_{2}, \quad e_{3} e_{3}=e_{3}, \quad e_{4} e_{3}=e_{4}$;

$A s_{4}^{50}: e_{1} e_{1}=e_{1}, \quad e_{1} e_{2}=e_{2}, \quad e_{1} e_{3}=e_{3}, \quad e_{1} e_{4}=e_{4}, \quad e_{2} e_{1}=e_{2}, \quad e_{2} e_{2}=e_{4}$,

$e_{4} e_{1}=e_{4}$

$A s_{4}^{51}: e_{1} e_{1}=e_{1}, \quad e_{1} e_{2}=e_{2}, \quad e_{1} e_{3}=e_{3}, \quad e_{1} e_{4}=e_{4}, \quad e_{2} e_{1}=e_{2}, \quad e_{3} e_{1}=e_{3}$, 


$$
A s_{4}^{52}: e_{1} e_{1}=e_{1}, \quad e_{1} e_{2}=e_{2}, \quad e_{1} e_{4}=e_{4}, \quad e_{2} e_{1}=e_{2}, \quad e_{2} e_{2}=e_{4}, \quad \begin{array}{r}
e_{4} e_{1}=e_{4} ; \\
e_{3} e_{1}=e_{3}, \\
e_{4} e_{1}=e_{4} ;
\end{array}
$$

$$
\begin{aligned}
& A s_{4}^{53}: e_{1} e_{1}=e_{1}, \quad e_{2} e_{2}=e_{2}, \quad e_{2} e_{3}=e_{3}, \quad e_{2} e_{4}=e_{4}, \quad e_{3} e_{2}=e_{3}, \quad e_{3} e_{3}=e_{4}, \\
& e_{4} e_{2}=e_{4} \text {; } \\
& A s_{4}^{54}: e_{1} e_{1}=e_{1}, \quad e_{1} e_{2}=e_{2}, \quad e_{2} e_{3}=e_{1}, \quad e_{2} e_{4}=e_{2}, \quad e_{3} e_{1}=e_{3}, \quad e_{3} e_{2}=e_{4}, \\
& e_{4} e_{3}=e_{3}, \quad e_{4} e_{4}=e_{4} \\
& A s_{4}^{55}: e_{1} e_{1}=e_{1}, \quad e_{1} e_{2}=e_{2}, \quad e_{1} e_{3}=e_{3}, \quad e_{1} e_{4}=e_{4}, \quad e_{2} e_{1}=e_{2}, \quad e_{2} e_{2}=e_{4}, \\
& e_{3} e_{1}=e_{3}, \quad e_{4} e_{1}=e_{4} \\
& A s_{4}^{56}: e_{1} e_{1}=e_{1}, \quad e_{1} e_{2}=e_{2}, \quad e_{1} e_{3}=e_{3}, \quad e_{1} e_{4}=e_{4}, \quad e_{2} e_{1}=e_{2}, \quad e_{2} e_{3}=e_{4}, \\
& e_{3} e_{1}=e_{3}, \quad e_{3} e_{2}=-e_{4}, \quad e_{4} e_{1}=e_{4} \\
& A s_{4}^{57}: e_{1} e_{1}=e_{1}, \quad e_{1} e_{2}=e_{2}, \quad e_{1} e_{3}=e_{3}, \quad e_{1} e_{4}=e_{4}, \quad e_{2} e_{1}=e_{2}, \quad e_{2} e_{2}=e_{3}, \\
& e_{2} e_{3}=e_{4}, \quad e_{3} e_{1}=e_{3}, \quad e_{3} e_{2}=e_{4}, \quad e_{4} e_{1}=e_{4} ; \\
& A s_{4}^{58}: e_{1} e_{1}=e_{1}, \quad e_{1} e_{2}=e_{2}, \quad e_{1} e_{3}=e_{3}, \quad e_{1} e_{4}=e_{4}, \quad e_{2} e_{1}=e_{2}, \quad e_{2} e_{2}=-e_{4}, \\
& e_{2} e_{3}=-e_{4}, \quad e_{3} e_{1}=e_{3}, \quad e_{3} e_{2}=e_{4}, \quad e_{4} e_{1}=e_{4} .
\end{aligned}
$$

In what follows, the following notations are used:

1. IC: isomorphism classes of algebras.

2. Dim: Dimensions of the algebra of derivations.

3. $m_{2}=d_{11}+d_{21}$,

4. $m_{3}=d_{33}-d_{11}$,

5. $m_{4}=\frac{2}{1-\alpha} d_{12}, \quad \alpha \neq 1$

6. $m_{5}=d_{22}+d_{33}$,

7. $m_{6}=d_{33}-d_{22}$.

The derivations of four-dimensional associative algebra are given as follows.

Theorem 14. The derivation of four-dimensional associative algebras has the following form: 
Table 1: Derivations of four-dimensional associative algebras

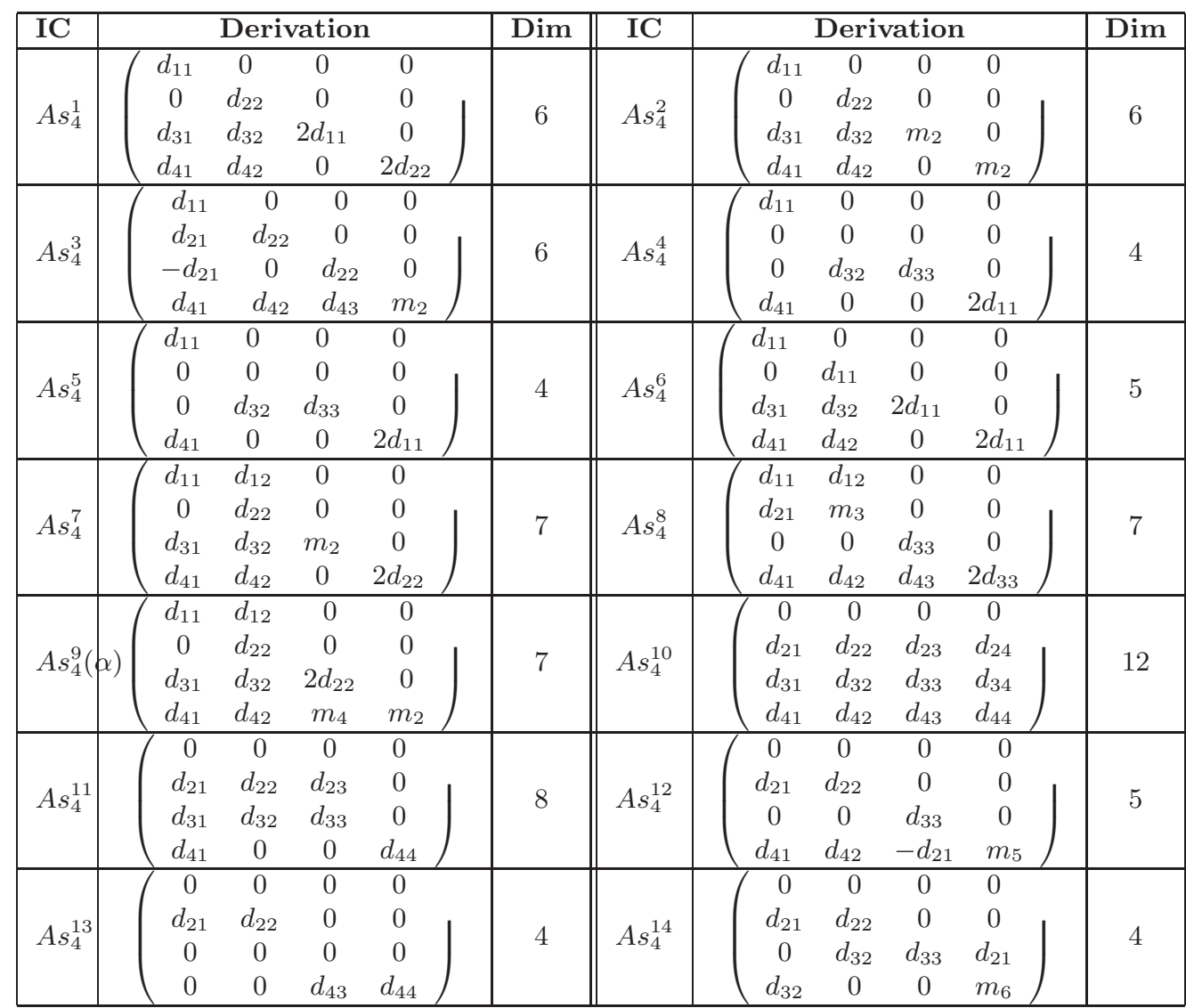

Continued to the next page 


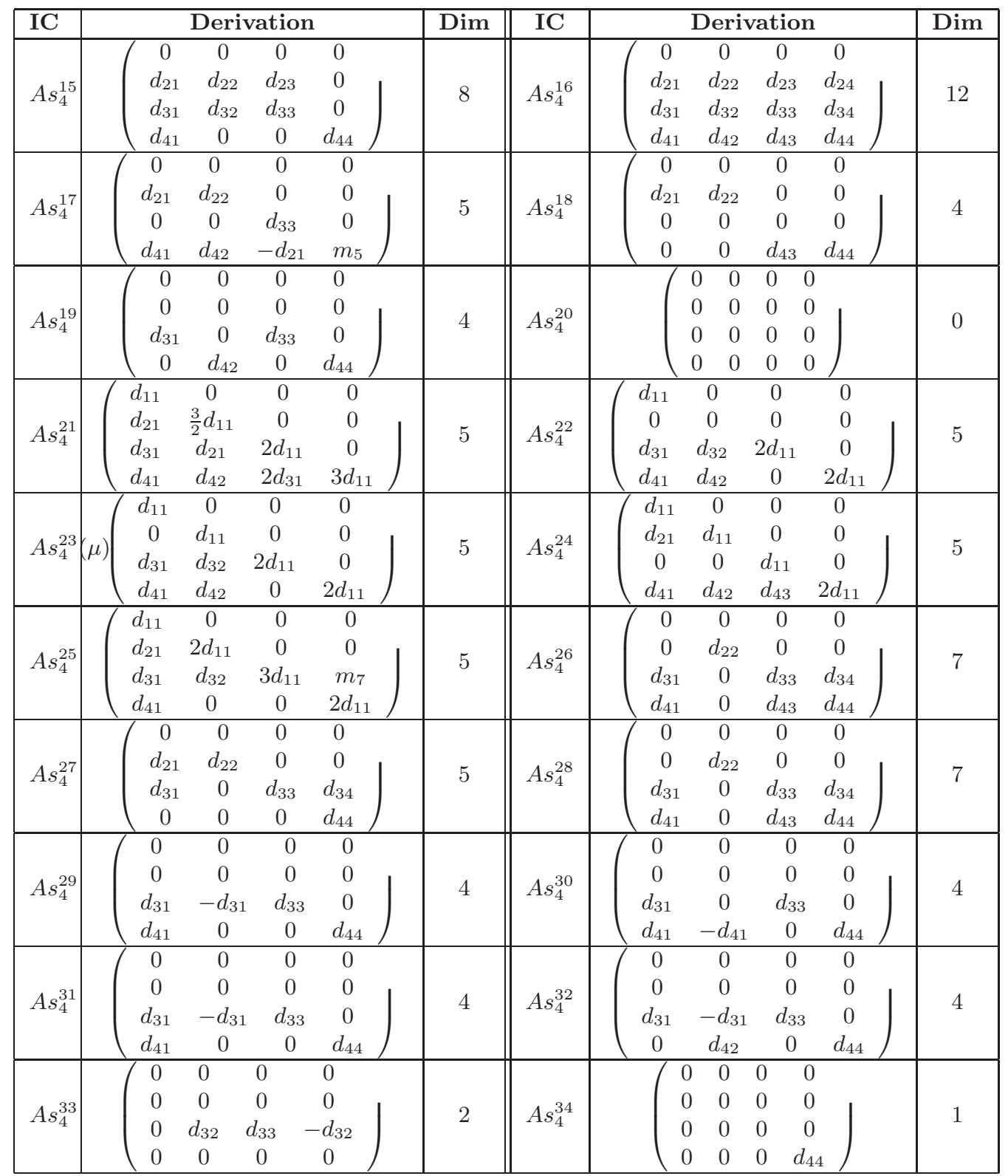

Continued to the next page 


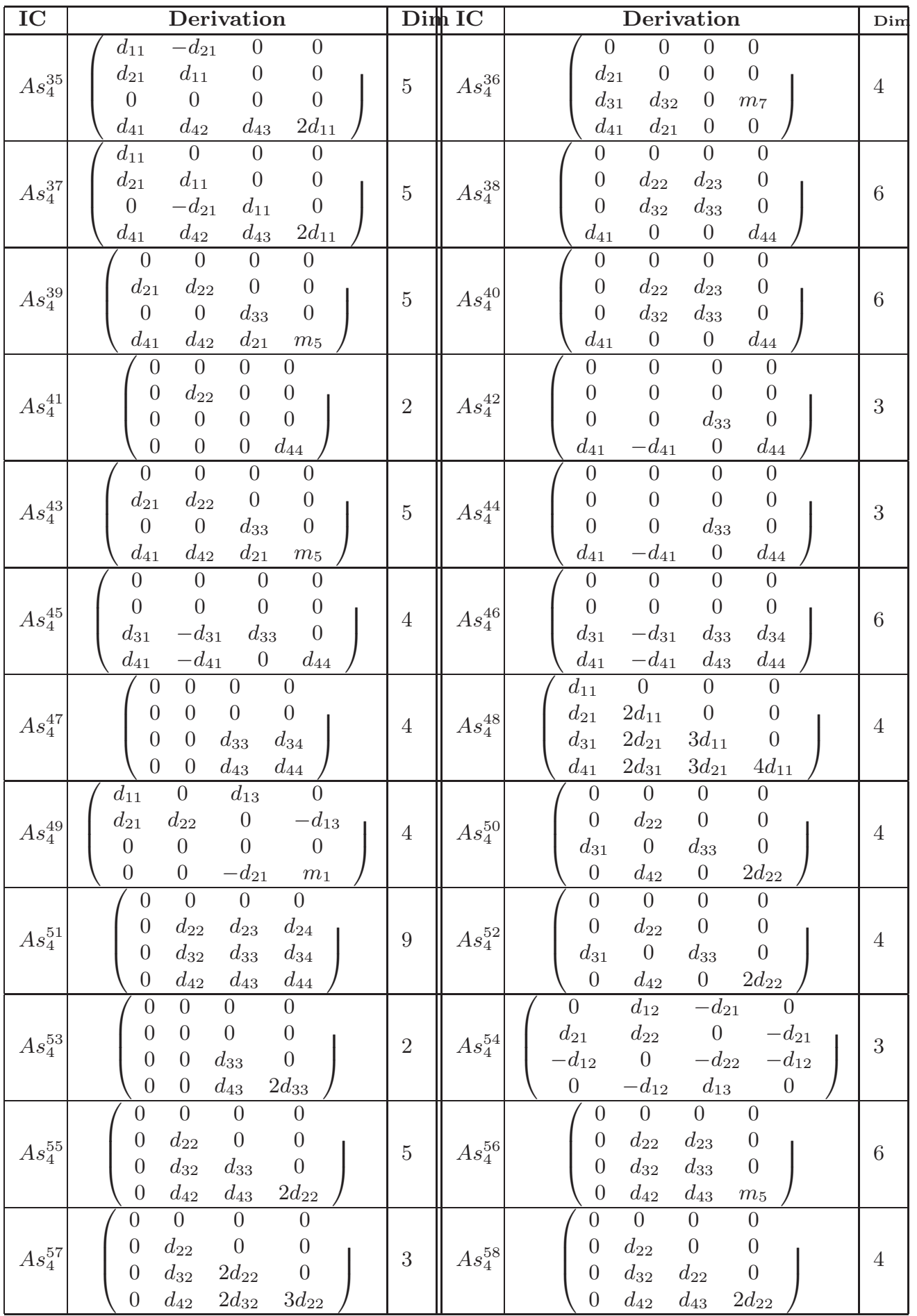


Proof. We provide the proof only for one case to illustrate the approach used, the other cases can be carried out similarly with small modification(s).

Let us consider $A s_{4}^{1}$. Applying the system of equation (1), we get $d_{12}=$ $d_{13}=d_{14}=d_{21}=d_{23}=d_{24}=d_{34}=d_{43}=0, d_{33}=2 d_{11}, d_{44}=2 d_{22}$. Therefore, the derivations for $A s_{4}^{1}$ are given by the matrices

$$
d=\left(\begin{array}{cccc}
d_{11} & 0 & 0 & 0 \\
0 & d_{22} & 0 & 0 \\
d_{31} & d_{32} & 2 d_{11} & 0 \\
d_{41} & d_{42} & 0 & 2 d_{22}
\end{array}\right)
$$

The dimension of the algebra $\operatorname{Der}\left(A s_{4}^{1}\right)$ of derivation of the class $A s_{4}^{1}$ is 6 .

\section{Remark 15.}

1. There is one class of characteristically nilpotent associative algebras in the list of isomorphism classes of four-dimensional associative algebras.

2. The dimensions of the derivation algebras in this case vary between zero and nine.

\section{Description of centroids of four dimensional associative algebras}

In this section, we give the description of centroids of four-dimensional complex associative algebras. Let $\left\{e_{1}, \ldots, e_{n}\right\}$ be a basis of a $n$-dimensional associative algebra $A$, then $e_{i} e_{j}=\sum_{k=1}^{n} \gamma_{i j}^{k} e_{k}$. The coefficients, $\left\{\gamma_{i j}^{k}\right\} \in \mathbb{C}^{n^{3}}$, of the above linear combinations are called the structure constants of $A$ on the basis $\left\{e_{1}, \ldots, e_{n}\right\}$. An element $\phi$ of the centroid $\Gamma(A)$, being a linear transformation of the vector space $A$, is represented in a matrix form $\left(a_{i j}\right)_{i, j=1,2, \cdots, n}$, i.e., $\phi\left(e_{i}\right)=\sum_{j=1}^{n} a_{j i} e_{j}$, $i=1,2, \cdots, n$. must satisfy the following systems of equations:

$$
\sum_{k=1}^{n}\left(\gamma_{i j}^{t} a_{k t}-a_{t i} \gamma_{t j}^{k}\right)=0
$$

and

$$
\sum_{k=1}^{n}\left(\gamma_{i j}^{k} a_{k t}-a_{t j} \gamma_{i t}^{k}\right)=0
$$


It is observed that if the structure constants $\left\{\gamma_{i j}^{k}\right\}$ of the associative algebra $A$ are given, then, in order to describe its centroid one has to solve the system of equations above with respect to the $a_{i j}, i, j=1,2, \ldots, n$. Again, we make use classification results of four-dimensional complex associative algebras from [10] to compute the centroids of four-dimensional complex associative algebras.

Theorem 16. The centroids of four dimensional complex associative algebras are given as follows:

Table 2: Centroids of four-dimensional associative algebras

\begin{tabular}{|c|c|c|c|c|c|c|c|c|c|c|c|}
\hline IC & \multicolumn{4}{|c|}{$\begin{array}{c}\text { Centroid } \\
\Gamma(A)\end{array}$} & Dim & IC & \multicolumn{4}{|c|}{$\begin{array}{c}\text { Centroid } \\
\Gamma(A)\end{array}$} & Dim \\
\hline \multirow{4}{*}{$A s_{4}^{1}$} & $a_{11}$ & 0 & 0 & 0 & \multirow{4}{*}{2} & \multirow{4}{*}{$A s_{4}^{2}$} & $\overline{a_{11}}$ & 0 & 0 & 0 & \multirow{4}{*}{5} \\
\hline & 0 & $a_{22}$ & 0 & 0 & & & 0 & $a_{11}$ & 0 & 0 & \\
\hline & 0 & 0 & $a_{11}$ & 0 & & & $a_{31}$ & $a_{32}$ & $a_{11}$ & 0 & \\
\hline & 0 & 0 & 0 & $a_{11}$ & & & $a_{41}$ & $a_{42}$ & 0 & $a_{11}$ & \\
\hline \multirow{4}{*}{$A s_{4}^{3}$} & $\left(a_{11}\right.$ & 0 & 0 & 0 & \multirow{4}{*}{4} & \multirow{4}{*}{$A s_{4}^{4}$} & $a_{11}$ & 0 & 0 & 0 & \multirow{4}{*}{3} \\
\hline & 0 & $a_{11}$ & 0 & 0 & & & 0 & $a_{22}$ & 0 & 0 & \\
\hline & 0 & 0 & $a_{11}$ & 0 & & & 0 & 0 & $a_{22}$ & 0 & \\
\hline & $a_{41}$ & $a_{42}$ & $a_{43}$ & $a_{11}$ & & & $a_{41}$ & 0 & 0 & $a_{11}$ & \\
\hline \multirow{4}{*}{$A s_{4}^{5}$} & $a_{11}$ & 0 & 0 & 0 & \multirow{4}{*}{3} & \multirow{4}{*}{$A s_{4}^{6}$} & $\overline{a_{11}}$ & 0 & 0 & 0 & \multirow{4}{*}{5} \\
\hline & 0 & $a_{22}$ & 0 & 0 & & & 0 & $a_{11}$ & 0 & 0 & \\
\hline & 0 & 0 & $a_{22}$ & 0 & & & $a_{31}$ & $a_{32}$ & $a_{11}$ & 0 & \\
\hline & $a_{41}$ & 0 & 0 & $a_{11}$ & & & $a_{41}$ & $a_{42}$ & 0 & $a_{11}$ & \\
\hline \multirow{4}{*}{$A s_{4}^{7}$} & $a_{11}$ & $a_{12}$ & 0 & 0 & \multirow{4}{*}{6} & \multirow{4}{*}{$A s_{4}^{8}$} & $a_{11}$ & 0 & 0 & 0 & \multirow{4}{*}{4} \\
\hline & 0 & $a_{11}$ & 0 & 0 & & & 0 & $a_{11}$ & 0 & 0 & \\
\hline & $a_{31}$ & $a_{32}$ & $a_{11}$ & $a_{12}$ & & & 0 & 0 & $a_{11}$ & 0 & \\
\hline & $a_{41}$ & $a_{42}$ & 0 & $a_{11}$ & & & $a_{41}$ & $a_{42}$ & $a_{43}$ & $a_{11}$ & \\
\hline \multirow{4}{*}{$A s_{4}^{9}(\alpha)$} & $a_{11}$ & 0 & 0 & 0 & \multirow{4}{*}{5} & \multirow{4}{*}{$A s_{4}^{10}$} & $\overline{a_{11}}$ & 0 & 0 & 0 & \multirow{4}{*}{4} \\
\hline & 0 & $a_{11}$ & 0 & 0 & & & $a_{21}$ & $a_{11}$ & 0 & 0 & \\
\hline & $a_{31}$ & $a_{32}$ & $a_{11}$ & 0 & & & $a_{31}$ & 0 & $a_{11}$ & 0 & \\
\hline & $a_{41}$ & $a_{42}$ & 0 & $a_{11}$ & & & $a_{41}$ & 0 & 0 & $a_{11}$ & \\
\hline \multirow{4}{*}{$A s_{4}^{11}$} & $\overline{a_{11}}$ & 0 & 0 & 0 & \multirow{4}{*}{1} & \multirow{4}{*}{$A s_{4}^{12}$} & $\overline{a_{11}}$ & 0 & 0 & 0 & \multirow{4}{*}{1} \\
\hline & 0 & $a_{11}$ & 0 & 0 & & & 0 & $a_{11}$ & 0 & 0 & \\
\hline & 0 & 0 & $a_{11}$ & 0 & & & 0 & 0 & $a_{11}$ & 0 & \\
\hline & 0 & 0 & 0 & $a_{11}$ & & & 0 & 0 & 0 & $a_{11}$ & \\
\hline \multirow{4}{*}{$A s_{4}^{13}$} & $a_{11}$ & 0 & 0 & 0 & \multirow{4}{*}{2} & \multirow{4}{*}{$A s_{4}^{14}$} & $\overline{a_{11}}$ & 0 & 0 & 0 & \\
\hline & 0 & $a_{11}$ & 0 & 0 & & & 0 & $a_{11}$ & 0 & 0 & 1 \\
\hline & 0 & 0 & $a_{33}$ & 0 & & & 0 & 0 & $a_{11}$ & 0 & 1 \\
\hline & 0 & 0 & 0 & $a_{33}$ & & & 0 & 0 & 0 & $a_{11}$ & \\
\hline
\end{tabular}

Continued to the next page 


\begin{tabular}{|c|c|c|c|c|c|c|c|c|c|c|c|}
\hline \multirow{5}{*}{$\begin{array}{c}\text { IC } \\
A s_{4}^{15}\end{array}$} & \multicolumn{4}{|c|}{$\begin{array}{c}\text { Centroid } \\
\Gamma(A)\end{array}$} & \multirow{5}{*}{$\begin{array}{c}\text { Dim } \\
1\end{array}$} & \multirow{5}{*}{$\begin{array}{c}\text { IC } \\
A s_{4}^{16}\end{array}$} & \multicolumn{4}{|c|}{$\begin{array}{c}\text { Centroid } \\
\Gamma(A)\end{array}$} & \multirow{5}{*}{\begin{tabular}{|c|} 
Dim \\
1 \\
\end{tabular}} \\
\hline & $a_{11}$ & 0 & 0 & 0 & & & $a_{11}$ & 0 & 0 & 0 & \\
\hline & 0 & $a_{11}$ & 0 & 0 & & & 0 & $a_{11}$ & 0 & 0 & \\
\hline & 0 & 0 & $a_{11}$ & 0 & & & 0 & 0 & $a_{11}$ & 0 & \\
\hline & 0 & 0 & 0 & $a_{11}$ & & & 0 & 0 & 0 & $\left.a_{11}\right)$ & \\
\hline \multirow{4}{*}{$A s_{4}^{17}$} & $a_{11}$ & 0 & 0 & 0 & \multirow{4}{*}{1} & \multirow{4}{*}{$A s_{4}^{18}$} & $a_{11}$ & 0 & 0 & 0 & \multirow{4}{*}{2} \\
\hline & 0 & $a_{11}$ & 0 & 0 & & & 0 & $a_{11}$ & 0 & 0 & \\
\hline & 0 & 0 & $a_{11}$ & 0 & & & 0 & 0 & $a_{33}$ & 0 & \\
\hline & 0 & 0 & 0 & $\left.a_{11}\right)$ & & & 0 & 0 & 0 & $\left.a_{33}\right)$ & \\
\hline \multirow{4}{*}{$A s_{4}^{19}$} & $a_{11}$ & 0 & 0 & 0 & \multirow{4}{*}{2} & \multirow{4}{*}{$A s_{4}^{20}$} & $a_{11}$ & 0 & 0 & 0 & \multirow{4}{*}{4} \\
\hline & 0 & $a_{22}$ & 0 & 0 & & & 0 & $a_{22}$ & 0 & 0 & \\
\hline & 0 & 0 & $a_{11}$ & 0 & & & 0 & 0 & $a_{33}$ & 0 & \\
\hline & 0 & 0 & 0 & $\left.a_{22}\right)$ & & & 0 & 0 & 0 & $a_{44}$ & \\
\hline \multirow{4}{*}{$A s_{4}^{21}$} & $a_{11}$ & 0 & 0 & 0 & \multirow{4}{*}{4} & \multirow{4}{*}{$A s_{4}^{22}$} & $a_{11}$ & 0 & 0 & 0 & \multirow{4}{*}{5} \\
\hline & 0 & $a_{11}$ & 0 & 0 & & & 0 & $a_{11}$ & 0 & 0 & \\
\hline & $a_{31}$ & 0 & $a_{11}$ & 0 & & & $a_{31}$ & $a_{32}$ & $a_{11}$ & 0 & \\
\hline & $a_{41}$ & $a_{42}$ & $a_{31}$ & $a_{11} /$ & & & $a_{41}$ & $a_{42}$ & 0 & $a_{11}$ & \\
\hline \multirow{4}{*}{$A s_{4}^{23}(\mu)$} & $a_{11}$ & 0 & 0 & 0 & \multirow{4}{*}{5} & \multirow{4}{*}{$A s_{4}^{24}$} & $a_{11}$ & 0 & 0 & 0 & \multirow{4}{*}{4} \\
\hline & 0 & $a_{11}$ & 0 & 0 & & & 0 & $a_{11}$ & 0 & 0 & \\
\hline & $a_{31}$ & $a_{32}$ & $a_{11}$ & 0 & & & 0 & 0 & $a_{11}$ & 0 & \\
\hline & $a_{41}$ & $a_{42}$ & 0 & $a_{11} /$ & & & $a_{41}$ & $a_{42}$ & $a_{4}$ & $a_{11}$ & \\
\hline \multirow{4}{*}{$A s_{4}^{25}$} & $a_{11}$ & 0 & 0 & 0 & & & $a_{11}$ & 0 & 0 & 0 & \\
\hline & $a_{21}$ & $a_{22}$ & 0 & $a_{24}$ & 7 & $A s^{26}$ & $a_{21}$ & $a_{11}$ & 0 & 0 & 4 \\
\hline & $a_{31}$ & $a_{32}$ & $a_{11}$ & 0 & & $\mathrm{AO}_{4}$ & $a_{31}$ & 0 & $a_{11}$ & 0 & \\
\hline & $a_{41}$ & 0 & 0 & $\left.a_{11}\right)$ & & & $a_{41}$ & 0 & 0 & $a_{11}$ & \\
\hline & $a_{11}$ & 0 & 0 & 0 & & & $a_{11}$ & 0 & 0 & 0 & \\
\hline$A s_{4}^{27}$ & 0 & $a_{11}$ & 0 & 0 & 2 & $A s_{4}^{28}$ & $a_{21}$ & $a_{11}$ & 0 & 0 & 2 \\
\hline$A s_{4}$ & 0 & 0 & $a_{11}$ & 0 & 2 & $A s_{4}$ & 0 & 0 & $a_{1}$ & 0 & 2 \\
\hline & $a_{41}$ & 0 & 0 & $\left.a_{11}\right)$ & & & 0 & 0 & 0 & $a_{11}$ & \\
\hline & $a_{11}$ & 0 & 0 & 0 & & & $a_{11}$ & 0 & 0 & 0 & \\
\hline$A s^{29}$ & 0 & $a_{11}$ & 0 & 0 & 1 & $A s^{30}$ & 0 & $a_{22}$ & 0 & 0 & 2 \\
\hline & 0 & 0 & $a_{11}$ & 0 & & & 0 & 0 & $a_{1}$ & 0 & \\
\hline & 0 & 0 & 0 & $\left.a_{11}\right)$ & & & 0 & 0 & 0 & $a_{11}$ & \\
\hline & $a_{11}$ & 0 & 0 & 0 & & & $a_{11}$ & 0 & 0 & 0 & \\
\hline$\Delta \mathrm{s}^{31}$ & 0 & $a_{11}$ & 0 & 0 & 1 & $4 \mathrm{~s}^{32}$ & 0 & $a_{11}$ & 0 & 0 & \\
\hline$A s_{4}^{0}$ & 0 & 0 & $a_{11}$ & 0 & 1 & $A s_{4}^{-1}$ & 0 & 0 & $a_{1}$ & 0 & 1 \\
\hline & 0 & 0 & 0 & $\left.a_{11}\right)$ & & & 0 & 0 & 0 & $a_{11}$ & \\
\hline & $a_{11}$ & 0 & 0 & 0 & & & $a_{11}$ & 0 & 0 & 0 & \\
\hline$A s^{33}$ & 0 & $a_{22}$ & 0 & 0 & 2 & $A s^{34}$ & 0 & $a_{22}$ & 0 & 0 & 1 \\
\hline & 0 & 0 & $a_{22}$ & 0 & 2 & $10_{4}$ & 0 & 0 & $a_{3}$ & 0 & 1 \\
\hline & 0 & 0 & 0 & $a_{22}$ & & & 0 & 0 & 0 & $a_{33}$ & \\
\hline
\end{tabular}

Continued to the next page 


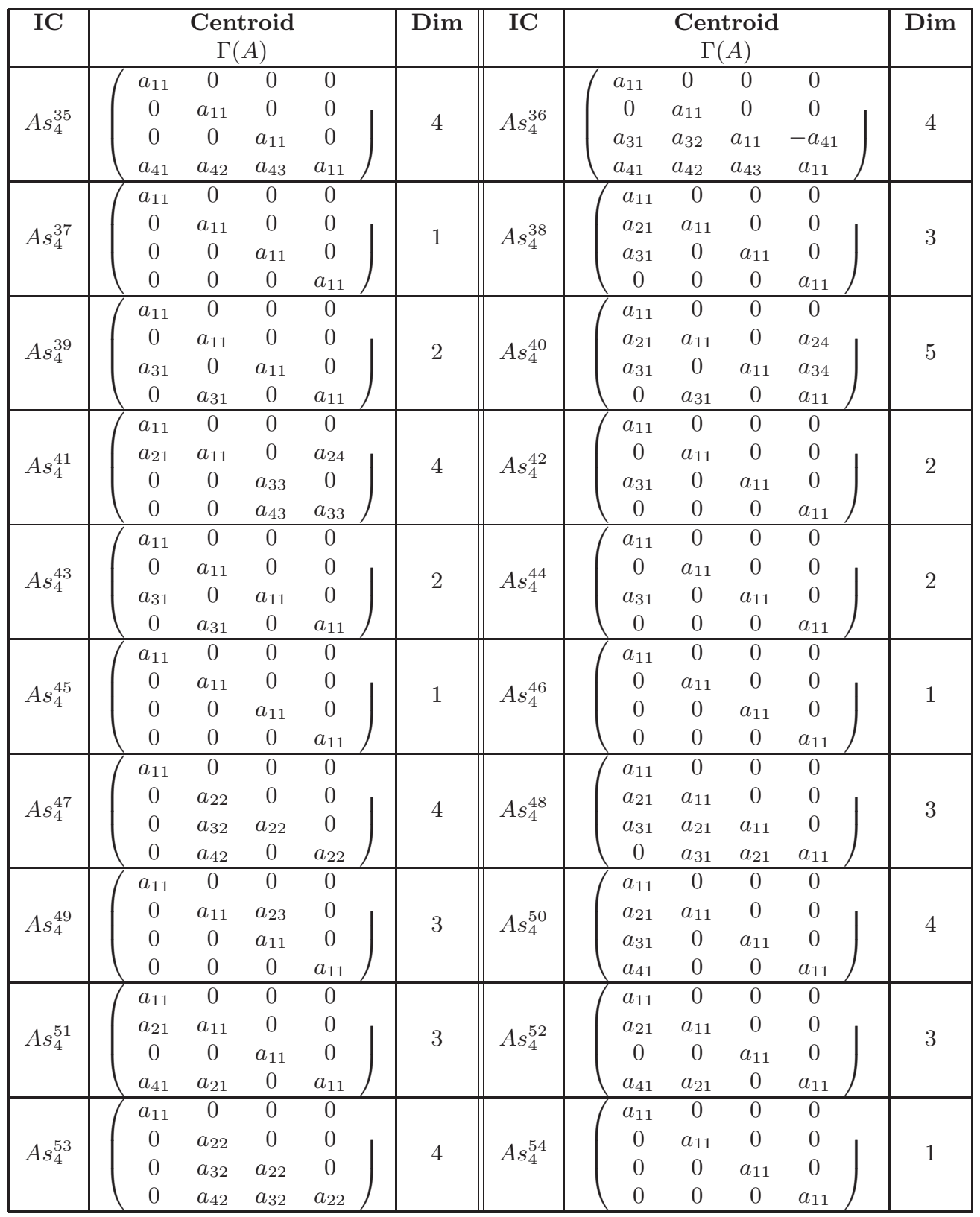

Continued to the next page 


\begin{tabular}{|c|c|c|c|c|c|c|c|c|c|c|c|}
\hline IC & \multicolumn{4}{|c|}{$\begin{array}{c}\text { Centroid } \\
\Gamma(A)\end{array}$} & $\overline{\text { Dim }}$ & IC & \multicolumn{4}{|c|}{$\begin{array}{c}\text { Centroid } \\
\Gamma(A)\end{array}$} & Dim \\
\hline \multirow{4}{*}{$A s_{4}^{55}$} & $a_{11}$ & 0 & 0 & 0 & \multirow{4}{*}{4} & \multirow{4}{*}{$A s_{4}^{56}$} & $a_{11}$ & 0 & 0 & 0 & \multirow{4}{*}{4} \\
\hline & $a_{21}$ & $a_{11}$ & 0 & 0 & & & $a_{21}$ & $a_{11}$ & 0 & 0 & \\
\hline & $a_{31}$ & 0 & $a_{1}$ & 0 & & & $a_{31}$ & 0 & $a_{11}$ & 0 & \\
\hline & $a_{41}$ & $a_{21}$ & 0 & $a_{11}$ & & & $a_{41}$ & $-a_{31}$ & $-a_{21}$ & $a_{11}$ & \\
\hline \multirow{4}{*}{$A s_{4}^{57}$} & $a_{11}$ & 0 & $\overline{0}$ & 0 & \multirow{4}{*}{2} & \multirow{4}{*}{$A s_{4}^{58}$} & $a_{11}$ & 0 & 0 & 0 & \multirow{4}{*}{2} \\
\hline & 0 & $a_{11}$ & 0 & 0 & & & 0 & $a_{11}$ & 0 & 0 & \\
\hline & 0 & 0 & $a_{1}$ & 0 & & & 0 & 0 & $a_{11}$ & 0 & \\
\hline & $a_{41}$ & 0 & 0 & $a_{11}$ & & & $a_{41}$ & 0 & 0 & $a_{11}$ & \\
\hline
\end{tabular}

Proof. Let us consider $A s_{4}^{1}$, the structure constants are given as follows $\gamma_{11}^{3}=1, \gamma_{22}^{4}=1$ others being zero. From the systems of equations (2) and (3), we have

$a_{12}=a_{13}=a_{14}=a_{21}=a_{23}=a_{24}=a_{31}=a_{32}=a_{34}=a_{41}=a_{42}=a_{43}=0$ and $a_{33}=a_{11}=a_{44}$. Therefore, we obtain the centroids of $A s_{4}^{1}$ in matrix form as follows:

$$
\Gamma\left(A s_{4}^{1}\right)=\left\{\left(\begin{array}{cccc}
a_{11} & 0 & 0 & 0 \\
0 & a_{22} & 0 & 0 \\
0 & 0 & a_{11} & 0 \\
0 & 0 & 0 & a_{11}
\end{array}\right) \mid a_{11}, a_{22} \in \mathbb{C}\right\} .
$$

The computations for the other classes are carried out similarly.

\section{Acknowledgments}

The authors acknowledge Universiti Putra Malaysia for the support by the grant GP-IPS/2014/9449300.

\section{References}

[1] N. Jacobson, A note on automorphisms and derivations of Lie algebras, Proc. Amer. Math. Soc., 6(2), (1955), 281-283.

[2] J. Dixmier, W.G. Lister, Derivations of nilpotent Lie algebras, Proc. Amer. Math. Soc., 8 (1957), 155-158.

[3] G. Leger, S. Togo, Characteristically nilpotent Lie algebras, Duke Math. J., 26(4), (1959), 623-628. 
[4] J. Tits, Sur les constantes de structure et le theorem dexistence des algebres de Lie semisimples, Publ. Math. I.H.E.S., 31(1), (1966), 21-58. http://dx.doi.org/10.1007/BF02684801.

[5] B. Pierce, Linear Associative algebras, Amer. Math. Journal, (1881), 4(1) 97-229. http://www.jstor.org/stable/2369153.

[6] O.C. Hazlett, On the classification and invariantive characterization of nilpotent algebras, American Journal of Mathematics, 38(2), (1916), 109-138. http://www.jstor.org/stable/2370262

[7] G. Mazzola, The algebraic and geometric classification of associative algebras of dimension five, Manuscripta Math., 27(1), (1979), 81-101. http://dx.doi.org/10.1007/BF01297739.

[8] G. Mazzola, Generic finite schemes and Hochschild cocycles, Commentarii Mathematici Helvetici, 55(1), (1980), 267-293 . http://dx.doi.org/10.1007/BF02566686.

[9] B. Poonen, Isomorphism types of commutative algebras of finite rank, Computational Arithmetic Geometry, 463(2008), 111-120.

[10] I.S. Rakhimov, I.M. Rikhsiboev, W. Basri, Complete lists of low dimensional complex associative algebras, (2009). arXiv:0910.0932v1 [math.RA].

[11] G. Benkart, E. Neher, The centroid of extended affine and root graded Lie algebras, Journal of Pure and Applied Algebra, 205(1), (2006), 117-145.

[12] D.J. Melville, Centroids of nilpotent Lie algebras, Comm. Algebra, 20(12), (1992), 36493682 .

[13] M.A. Fiidow, I.S. Rakhimov, S.K. Said Hussain, Derivations and Centroids of Associative algebras. IEEE Proceedings of International Conference on Research and Education in Mathematics (ICREM7). (2015), 227-232. http://dx.doi.org/10.1109/ICREM.2015.7357059. 
